Original paper

\title{
Clinical aspects and treatment of hepatocellular carcinoma in north-eastern Poland
}

\author{
Tadeusz Wojciech Łapiński', Aleksandr Tarasik1,2, Marcin Januszkiewicz², Robert Flisiak \\ 'Department of Infectious Diseases and Hepatology, Medical University of Bialystok, Poland \\ ${ }^{2}$ Department of Oncological Surgery, Marie Curie-Sklodowska Oncology Centre in Bialystok, Poland
}

\begin{abstract}
Aim of the study: Hepatocellular carcinoma (HCC) is the most common type of primary liver cancer, with poor treatment outcomes often because of delayed diagnosis. The aim of this study was to assess the co-incidence of cirrhosis, alcohol abuse, hepatitis B virus (HBV) or hepatitis C virus (HCV) infection and fatty liver disease in patients in the population of north-eastern Poland, to analyse the usefulness of $\alpha$-fetoprotein (AFP) in the diagnosis of HCC and to assess the effectiveness of HCC treatment in this group.

Material and methods: The study involved 104 patients diagnosed with HCC. The age, sex, comorbidities, HCC risk factors and levels of AFP were analysed. The effect of antiviral therapy of HCV and HBV on HCC development was observed and the effectiveness of therapies used in the treatment of HCC was assessed.

Results: Over $90 \%$ of patients with $\mathrm{HCC}$ were older than 45 years. The incidence of $\mathrm{HCC}$ was higher in men than in women. Patients with $\mathrm{HCC}$ were also diagnosed with cirrhosis $(72 \%)$, alcohol abuse (35\%), HCV infection (35\%), HBV infection (24\%), and fatty liver disease (13\%). HCC developed in 9/25 (36\%) patients positive for HBV despite effective antiviral therapy. The highest AFP levels were found in HBV-positive patients. The mean survival time was 19 months for partial hepatectomy and 16 months for thermal ablation.

Conclusions: The main predisposing factor for HCC is cirrhosis, followed by alcohol abuse and infection with HCV. Effective antiviral therapy for HBV does not prevent the development of HCC in all cases. Since $29 \%$ of patients were disqualified from HCC treatment due to an advanced stage of cancer, it indicates insufficient screening for HCC. Partial hepatectomy and radiofrequency ablation show comparable effectiveness in the treatment of HCC.
\end{abstract}

Key words: treatment, HCC, clinical aspects, AFP.

Address for correspondence:

Dr. Aleksandr Tarasik, Department of Infectious Diseases and Hepatology, Medical University of Bialystok, Poland, e-mail: olek.tarasik@gmail.com

\section{Introduction}

Hepatocellular carcinoma (HCC) is the most common $(70 \%)$ type of primary liver cancer. Recent epidemiological studies conducted in the United States revealed a 4 -fold increase in the incidence of HCC over the last 40 years $(4.8 / 100,000$ people in 2005 2007), and its incidence is expected to increase to $8.8 / 100,000$ [1]. The highest incidence of HCC was reported from Asia and Africa. The risk of developing HCC in the Polish population, as in most European countries, remains at a low level, and its incidence ac- cording to data reported by the World Health Organization (WHO) is 3.2/100,000 people. According to the Polish National Cancer Registry, HCC is diagnosed in approx. 2-2,500 people/year, and the annual mortality for HCC is approx. 2,500 per general population. These statistics indicate unsatisfactory detection and treatment of HCC. Major risk factors for developing $\mathrm{HCC}$ include cirrhosis, infection with $\mathrm{HBV}$ or HCV, and fatty liver disease. Other risk factors include the toxic effects of alcohol and aflatoxin, adenomas induced by hormonal drugs (contraception, anabolics), and metabolic liver diseases (especially haemochro- 
matosis, associated with a 300-fold increase in risk). HCC is usually diagnosed in cirrhotic patients. It is a heterogeneous tumour characterized by a complex mechanism of development and formation associated with genetic factors regulated by the activation or suppression of cellular oncogenes [2]. The survival of patients with HCC who are not qualified for cancer treatment is usually no longer than several months. The treatment plan for patients with HCC should consider recommendations included in the Barcelona clinic liver cancer (BCLC) staging system, clinical measures of the liver disease (the Child-Pugh score), and the patient's general well-being (ECOG system). Patients with HCC are treated by partial hepatectomy, ablation, liver transplant and systemic therapy. Nevertheless, combined therapy involving more than one modality is becoming increasingly popular.

The aim of this study was to assess the incidence of cirrhosis, alcohol abuse, hepatitis B virus (HBV) or hepatitis $\mathrm{C}$ virus (HCV) infection and fatty liver disease in patients diagnosed with HCC in the population of north-eastern Poland. The usefulness of $a$-fetoprotein (AFP) in the diagnosis of HCC was analysed in relation to the stage and grade of cancer, and coexisting predictive factors for HCC. The effectiveness of therapies used in the treatment of HCC was assessed.

\section{Material and methods}

The severity of liver damage was determined by morphological examination of a liver biopsy specimen, or analysis by means of shear wave elastography (SWE, Aixplorer, Supersonic Imagine, France) or transient elastography (TE, FibroScan Echosense, France). The pathomorphological assessment of cirrhosis and hepatitis relied on the Scheuer classification ${ }^{1}$ (cirrhosis and hepatitis graded 0 to 4). Cirrhosis in SWE or TE was measured in kilopascals $(\mathrm{kPa})$, and expressed in a $0-4$ scoring system, which corresponds to the METAVIR histological scale recommended by the manufacturer and comparable to the Scheuer classification. Infection with HBV was confirmed by detecting the HBs antigen in serum using the micro-enzyme immunoassay (MEIA) and dedicated kits (Abbott, Germany). The concentration of HBV-DNA in serum was analysed by means of RT-PCR and the COBAS AmpliPre/COBAS TagMan HBV Test, version 2.0 (Roche, Germany).
HCV-RNA was quantified using RT-PCR and the COBAS HCV Test, version 2.0 (Roche, Germany). $\mathrm{HCV}$ genotypes were identified by electrophoretic amplification. Electropherograms were visualized in the system for documentation and image analysis (UVI-KS400i/Image PC, Syngen Biotech, USA) ${ }^{2}$.

Alcohol abuse was defined as at least a 2-year-long period of alcohol consumption causing mental and physical addiction.

Serum levels of AFP were measured using a COBAS 6000 apparatus and chemiluminescence tests (Roche, Germany) ${ }^{3}$.

HCC was treated by thermal ablation using the Cool-tip TM RF Ablation System, E Series (Covidien, Switzerland). Single active electrodes with a $3 \mathrm{~cm}$-long active part and a total length of $10-25 \mathrm{~cm}$ were used.

An ultrasonic shear (SonoSurg, Olympus, Japan) was used to dissect the liver parenchyma during partial hepatectomy. Ultrasonically fragmented liver tissue was removed by a suction module, which allowed for the precise dissection of the liver parenchyma and saving vascular structures.

Smaller vessels in the liver parenchyma were sealed using a ThunderBeat electrosurgical generator (Olympus, Japan) or a harmonic knife (Johnson \& Johnson, USA).

The study protocol was approved by the Bioethical Committee of the Medical University in Białystok (R-I-002/395/2018).

\section{Statistical analysis}

Data were processed using STATISTICA.PL software (StatSoft). The Mann-Whitney $U$ test, Spearman test, Student's $t$-test and chi-square test $\left(\chi^{2}\right)$ were used for statistical analysis. Results were presented as means $(\mathrm{x})$ and their standard errors (SE). The level of significance was adopted at $p<0.05$.

\section{Results}

Hepatocellular carcinoma was diagnosed in 104 patients from north-eastern Poland over a period of 8 years (2012-2019). Over 90\% of patients with HCC were older than 45 years. The incidence of HCC was higher in men than in women. Cirrhosis was diagnosed in $72 \%$ of patients. The most common factors

\footnotetext{
${ }^{1}$ The morphology of biopsy specimens was assessed by Prof. A. Panasiuk from the Department of Infectious Diseases and Hepatology, Medical University of Białystok.

${ }^{2}$ Analysis performed at the Department of Molecular Biology, Medical University of Białystok, head: Prof. J. Nikliński.

${ }^{3}$ Analysis performed at the Department of Medical Diagnostics, Teaching Hospital in Białystok, ul. Żurawia 14, head: mgr K. Jarocka.
} 
associated with the risk of $\mathrm{HCC}$, irrespective of cirrhosis, were infection with $\mathrm{HCV}$ and alcohol abuse.

HCC developed in 9/25 (36\%) patients positive for HBV despite effective antiviral therapy. Tests of serum samples from these patients were negative for HBVDNA. HBV viral load was high in the other 16 patients (HBV-DNA $\geq 4 \log _{10} \mathrm{IU} / \mathrm{ml}$ ).

HCC developed in 3/36 (36\%) patients positive for HCV despite effective antiviral therapy. Patients positive for HCV were identified with genotype $1 \mathrm{~b}(n=30,83 \%)$, genotype $3 \mathrm{a}(n=4,11 \%)$, or genotype $4(n=2,6 \%)$.

In most cases HCC was located in the right liver lobe, in segments VI, VII and VIII. Initially, 37\% of patients were diagnosed with 2-3 focal lesions larger than $5 \mathrm{~cm}$.

Distant metastases from $\mathrm{HCC}$ were found in the lungs $(n=6)$, lymph nodes of the hepatoduodenal ligament $(n=11)$, and in mediastinal lymph nodes $(n=3)$. Portal vein tumour thrombosis was diagnosed in 13 patients.

Elevated serum levels of AFP were found most frequently in patients positive for $\mathrm{HBV}$ and in patients with portal vein tumour thrombosis. There were no significant differences in the levels of AFP in relation to the size of the tumour, and possible metastases.

Partial hepatectomy and radiofrequency ablation were the most often used methods of HCC treatment in our study.

\section{Discussion}

Consistently with other reports, our study revealed significantly higher incidence of HCC in men than women ( $75 \%$ vs. $25 \%)$, and in patients aged over 45 years $(88 \%)$ [1]. Cirrhosis is the most serious risk factor for HCC, which is also consistent with global data. In our study, cirrhosis as an independent risk factor for HCC was diagnosed in $72 \%$ of patients. However, $84 \%$ of patients with HCC were cirrhotic and positive for $\mathrm{HBV}$, while $69 \%$ of patients were positive
Table 1. Characteristics of patients treated for hepatocellular carcinoma

\begin{tabular}{|c|c|c|}
\hline Variable & $n(\%)$ & $p$ \\
\hline \multicolumn{3}{|l|}{ Sex } \\
\hline Women & $26(25 \%)$ & $<0.001$ \\
\hline Men & $78(75 \%)$ & \\
\hline \multicolumn{3}{|l|}{ Age (years), mean (range) } \\
\hline Women & $68(35-82)$ & $>0.5$ \\
\hline Men & $63(30-87)$ & \\
\hline Patients older than 45 years & $95(91)$ & \\
\hline Women & $25(96)$ & $<0.001$ \\
\hline Men & $70(90)$ & \\
\hline \multicolumn{3}{|l|}{ Comorbidities } \\
\hline Alcoholic cirrhosis & $28(27)$ & \\
\hline Infection with HBV & $25(24)$ & \\
\hline Post-inflammatory cirrhosis & $21(84)$ & \\
\hline Infection with HCV & $36(35)$ & \\
\hline Post-inflammatory cirrhosis & $25(69)$ & \\
\hline Infection with HCV/HIV & $1(3)$ & \\
\hline Fatty liver disease & $14(13)$ & \\
\hline Primary biliary cirrhosis & $1(1)$ & \\
\hline Cirrhosis & $75(72)$ & $<0.001$ \\
\hline Alcohol abuse & $36(35)$ & \\
\hline
\end{tabular}

for HCV (the difference was not significant). Guro et al. and Ding et al. reported even higher incidence of cirrhosis (80-90\%) in patients with HCC $[3,4]$.

Alcoholic cirrhosis was diagnosed in $27 \%$ of patients with HCC, but in total $35 \%$ of patients with this cancer abused alcohol. Importantly, alcohol consumption is an independent predictor of HCC. Alcohol abuse, smoking and diabetes are increasingly often reported as risk factors for HCC [5].

The association between infection with HBV and HCC is well known. However, the fact that HCC is di-

Table 2. Selected clinical parameters of hepatocellular carcinoma $(\mathrm{HCC})$ in patients positive for HBV depending on antiviral treatment.

\begin{tabular}{lccc}
\hline & $\begin{array}{c}\text { Antiviral treatment } \\
\boldsymbol{n}=\mathbf{9}\end{array}$ & $\begin{array}{c}\text { No antiviral treatment } \\
\boldsymbol{n}=16\end{array}$ & $\boldsymbol{p}$ \\
\hline Age (years), mean & 68 & 60 & 0.4 \\
\hline $\begin{array}{l}\text { HBV-DNA viral load } \\
\text { mean (IU/ml) }\end{array}$ & 0 & $\begin{array}{c}145,908,539 \\
\text { range: } 7670-425,561,954\end{array}$ & 0.021 \\
\hline $\begin{array}{l}\text { Mean AFP } \\
\text { (ng/ml) }\end{array}$ & 790 & 9670 & 0.2 \\
\hline Mean number of tumours & range: $4-4,241$ & 2.3 & 0.4 \\
\hline Mean size of tumour $(\mathrm{mm})$ & 1.7 & 86 & $3-60,500$ \\
\hline Number of patients with diffuse HCC & 67 & $3(19 \%)$ & \\
\hline
\end{tabular}


Table 3. Location of hepatocellular carcinoma (HCC), number and size of tumours

\begin{tabular}{lc}
\hline Variable & $n(\%)$ \\
\hline Liver segment & $2(1)$ \\
\hline I & $22(11)$ \\
\hline V & $18(9)$ \\
\hline VI & $25(14)$ \\
\hline VII & $20(10)$ \\
\hline VIII & $38(20)$ \\
\hline Diffuse HCC & $39(20)$ \\
\hline Single tumour & $28(15)$ \\
\hline$\leqslant 5 \mathrm{~cm}$ & $12(12)$ \\
\hline$>5 \mathrm{~cm}$ & $28(27)$ \\
\hline$\leqslant 3$ tumours with the diameter & $10(10)$ \\
\hline$\leqslant 5 \mathrm{~cm}$ & $7(7)$ \\
\hline$>5 \mathrm{~cm}$ & $40(37)$ \\
\hline More than 3 tumours with the diameter & $7(7)$ \\
\hline$\leqslant 5 \mathrm{~cm}$ & 0 \\
\hline$>5 \mathrm{~cm}$ & \\
\hline
\end{tabular}

agnosed in patients who had effective antiviral therapy is very disturbing. Studies identified the following risk factors for the occurrence of HCC in patients who had successful antiviral treatment: older age, male sex, pre-existing cirrhosis, lower platelet counts, and higher hepatitis B core-related antigens. The annual incidence rate of HCC during nucleotide analogues (NA) treatment was $0.3-1.2 \%$ in non-cirrhotic patients and $1.8-6.0 \%$ in cirrhotic patients [6]. This may suggest that treatment with nucleoside or NA in some patients does not sufficiently suppress carcinogenesis. A retro-
Table 4. Patients with elevated $\alpha$-fetoprotein (AFP) levels

\begin{tabular}{lcc}
\hline Variable & $\begin{array}{c}\text { Level of AFP } \\
\text { (ng/ml), mean } \\
\text { (range) }\end{array}$ & $\begin{array}{c}\text { Patients with } \\
\text { AFP }>10 \times \\
\text { normal range }\end{array}$ \\
\hline Patients with cirrhosis, $n=75(72 \%)$ & $5835(4-132,459)$ & $41(55 \%)$ \\
\hline $\begin{array}{l}\text { Patients without cirrhosis, } n=29 \\
(28 \%)\end{array}$ & $1011(2-20,000)$ & $8(28 \%)$ \\
\hline Alcoholic cirrhosis, $n=28(26 \%)$ & $6495(3-132,459)$ & $13(46 \%)$ \\
\hline HCV-positive, $n=36(35 \%)$ & $3244(3-56,851)$ & $17(47 \%)$ \\
\hline HBV-positive, $n=25(24 \%)$ & $6710(4-60,500)$ & $18(72 \%)$ \\
\hline Fatty liver disease, $n=14(13 \%)$ & $51(2-423)$ & $1(7 \%)$ \\
\hline $\begin{array}{l}\text { Focal lesions with diameter } \leqslant 5 \mathrm{~cm}, \\
n=35\end{array}$ & $2149(2-56,851)$ & $16(46 \%)$ \\
\hline $\begin{array}{l}\text { Focal lesions with diameter }>5 \mathrm{~cm}, \\
n=69\end{array}$ & $5802(3-132,459)$ & $31(45 \%)$ \\
\hline Metastatic HCC, $n=16$ & $4183(2-60,500)$ & $5(31 \%)$ \\
\hline Non-metastatic HCC, $n=88$ & $4584(2-132,459)$ & $44(50 \%)$ \\
\hline $\begin{array}{l}\text { HCC with portal vein tumour } \\
\text { thrombosis, } n=13\end{array}$ & 12538 & $8(62 \%)$ \\
\hline $\begin{array}{l}\text { HCC without portal vein tumour } \\
\text { thrombosis, } n=91\end{array}$ & $3307(2-60,500)$ & $41(45 \%)$ \\
\hline & & \\
\hline
\end{tabular}

spective study by Liu indicated that nucleotide or nucleoside analogue therapy was associated with milder symptoms, fewer and smaller neoplastic lesions on imaging tests, and lower incidence of portal vein tumour thrombosis [7]. Our study also showed that patients successfully treated with antiviral agents and diagnosed with HCC had lower AFP levels, as well as fewer and smaller tumours. However, these observations may be attributed to closer monitoring of patients on antiviral therapy and thus earlier diagnosis of HCC.

The frequency of genotypes in our patients with HCC and positive for HCV was comparable to the frequency of genotypes in the general population of patients infected with HCV. The majority of HCC cases

Table 5. Effectiveness of different types of therapies in patients with hepatocellular carcinoma (HCC)

\begin{tabular}{lcccc}
\hline Type of therapy & $n(\%)$ & Survival time in months (range) & Mean survival time & Number of deaths (\%) \\
\hline Conservative treatment & $30(29)$ & $2-24$ & 7.6 & $25(83)$ \\
\hline Sorafenib & $22(21)$ & $3-36$ & 13.4 & $3(14)$ \\
\hline Thermal ablation ${ }^{*}$ & $22(21)$ & $8-36$ & 16.1 & $2(9)$ \\
\hline Partial hepatectomy ${ }^{8 \#}$ & $25(24)$ & $4-60$ & 19.3 & $2(8)$ \\
\hline Liver transplant\# & $2(2)$ & 50 & 22.6 & $2(67)$ \\
\hline Other modalities* & $3(3)$ & $12-38$ & & 34 \\
\hline Total & $104(100)$ & & & 0 \\
\hline
\end{tabular}


in our study were connected with HCV genotype $1 \mathrm{~b}$. The connection between type $1 \mathrm{~b}$ of the virus and HCC development was confirmed in the literature $[8,9]$. However, other researchers suggest an association between HCV genotypes 3 and higher incidence of HCC [10]. So, literature data on this theme are controversial and inconclusive.

Most patients with HCC present with 1 to 3 focal lesions [11], which is also consistent with our observations. In most patients tumours were located in the right liver lobe, in segments VI, VII and VIII. The same tumours' distribution in the liver parenchyma was observed by authors from China; in $57 \%$ of cases HCC was localized in the right lobe [12]. This location is difficult to explain, but it might be associated with the larger volume of the right liver lobe and the hepatic vasculature. Probably, better supply of blood stimulates cell division, which may promote the formation and development of tumour cells in the cirrhotic liver.

Our study found a lower incidence of distant metastases from HCC (15\%) compared to data in the literature. However, all researchers have emphasized that metastases from HCC are relatively rare compared to other cancers, which remains an interesting fact [13]. These observations are very important from the clinical and therapeutic point of view.

AFP is a useful marker in the diagnosis of HCC. However, it should never be a decisive test because of the histochemical diversity of primary liver cancers. Some of these tumours do not secrete AFP at all [14]. The analysis of the study population revealed no correlation between the AFP level and the number or total size of hepatic tumours. The highest levels of AFP were measured in patients positive for $\mathrm{HBV}$, patients with diffuse HCC, and those with portal vein tumour thrombosis. In addition, in HBV-positive patients, AFP levels in patients with replicating HBV were significantly higher compared to patients without a detectable viral load. These observations are already used for the assessment of aggressiveness in HCC [15].

Liver transplant is the most effective treatment for HCC. Although the number of patients treated with liver transplant in the study group was small, their survival was the longest. Unfortunately, the growing number of patients with HCC does not make liver transplant possible in all patients who qualify for this procedure. Therefore, alternative treatment methods are being improved.

Currently, partial hepatectomy and tumour resection is a standard therapy in patients with resectable HCC and sufficient liver function. In our study patients with HCC were treated by partial hepatectomy (24\%) and thermal ablation (21\%). The mean survival time in patients undergoing partial hepatectomy was 19.3 months, and was 3 months longer compared to patients treated with thermal ablation. Meta-analyses of studies on therapies used for HCC and conducted consistently with Cochrane criteria revealed no significant advantage of hepatectomy over thermal ablation, provided that patients were correctly qualified for relevant procedures $[16,17]$.

Systemic targeted therapy with sorafenib was associated with a mean 5.8 month longer survival compared to patients who received symptomatic treatment. Importantly, sorafenib combined with thermal ablation or partial hepatectomy did not improve the overall treatment success measured by longer survival time [18].

About $29 \%$ of patients with HCC in our study were disqualified from surgical treatment due to the advanced stage of cancer or cirrhosis. This rate is very high compared to statistics for other countries in Western Europe, where it was estimated at about 8\% [17]. The mean survival time of patients disqualified from treatment was 7.6 months, which is comparable to literature data. Symptomatic treatment in these patients has a significant effect on the quality of life and may extend the patient's survival to several months [19].

\section{Conclusions}

The main predisposing factor for HCC is cirrhosis, followed by alcohol abuse and HCV infection. Effective antiviral therapy for HBV lowers the risk of HCC development, but does not prevent it in all patients. Since $29 \%$ of patients were disqualified from HCC treatment due to an advanced stage of cancer, it indicates insufficient screening for HCC. Partial hepatectomy (anatomical and nonanatomical) and radiofrequency ablation (percutaneous and intraoperative) show comparable effectiveness in the treatment of HCC.

\section{Disclosure}

The authors declare no conflict of interest.

\section{References}

1. Massarweh NN, El-Serag HB. Epidemiology of hepatocellular carcinoma and intrahepatic cholangiocarcinoma. Cancer Control 2017; 24: 1073274817729245.

2. Golabi P, Fazel S, Otgonsuren M, et al. Mortality assessment of patients with hepatocellular carcinoma according to underlying disease and treatment modalities. Medicine (Baltimore) 2017; 96: e5904.

3. Guro H, Cho YJ, Han HS, et al. Current status of laparoscopic liver resection for hepatocellular carcinoma. Clin Mol Hepatol 2016; 22: 212-218. 
4. Ding WZ, Han GY, Jin HH, et al. Anti-IL-20 monoclonal antibody suppresses hepatocellular carcinoma progression Oncol Lett 2018; 16: 6156-6162.

5. Shen Y, Risch H, Lu L, et al. Risk factors for hepatocellular carcinoma (HCC) in the northeast of the United States: results of a case-control study. Cancer Causes Control 2020; 31: 321-332.

6. Hiramatsu N, Yamada R, Takehara T. The suppressive effect of nucleos $(t)$ ide analogue treatment on the incidence of hepatocellular carcinoma in chronic hepatitis B patients. J Gastroenterol Hepatol 2016; 31: 546-552.

7. Liu L. Clinical features of hepatocellular carcinoma with hepatitis B virus among patients on Nucleos(t)ide analog therapy. Infect Agent Cancer 2020; 15: 8.

8. Lee MH, Yang HI, Lu SN, et al. Hepatitis $\mathrm{C}$ virus genotype $1 \mathrm{~b}$ increases cumulative lifetime risk of hepatocellular carcinoma. Int J Cancer 2014; 135: 1119-1126.

9. Bruno S, Crosignani A, Maisonneuve P, et al. Hepatitis $C$ virus genotype $1 \mathrm{~b}$ as a major risk factor associated with hepatocellular carcinoma in patients with cirrhosis: a seventeen-year prospective cohort study. Hepatology 2007; 46: 1350.

10. Sukowati CH, El-Khobar KE, Ie SI, et al. Significance of hepatitis virus infection in the oncogenic initiation of hepatocellular carcinoma. World J Gastroenterol 2016; 22: 1497-1512.

11. Choi J, Kim GA, Han S, et al. Longitudinal assessment of three serum biomarkers to detect very early-stage hepatocellular carcinoma. Hepatology 2019; 69: 1983-1994.

12. Xiang L, Xiao L, Li J, et al. Safety and feasibility of laparoscopic hepatectomy for hepatocellular carcinoma in the posterosuperior liver segments. World J Surg 2015; 39: 1202-1209.

13. Farinati F, Vitale A, Spolverato G, et al.; ITA.LI.CA study group. Development and validation of a new prognostic system for patients with hepatocellular carcinoma. PLoS Med 2016; 13: e1002006.

14. Carr BI, Guerra V, Giannini EG, et al. A liver index and its relationship to indices of HCC aggressiveness. J Integr Oncol 2016; 5: 178.

15. Fang Y, Chen W, Liang X, et al. Comparison of long-term effectiveness and complications of radiofrequency ablation with hepatectomy for small hepatocellular carcinoma. J Gastroenterol Hepatol 2014; 29: 193-200.

16. Majumdar A, Roccarina D, Thorburn D, et al. Management of people with early- or very early-stage hepatocellular carcinoma: an attempted network meta-analysis. Cochrane Database Syst Rev 2017; 3: CD011650.

17. Giannini EG, Farinati F, Ciccarese F, et al.; Italian Liver Cancer (ITA.LI.CA) group. Prognosis of untreated hepatocellular carcinoma. Hepatology 2015; 61: 184-190.

18. Jackson R, Psarelli E, Berhane S, Johnson PJ. Predictors of sorafenib benefit in patients with hepatocellular carcinoma. J Hepatol 2018; 68: 619-620.

19. Llovet JM, Ricci S, Mazzaferro V, et al., for the SHARP Investigators Study Group. Sorafenib in advanced hepatocellular carcinoma. N Engl J Med 2008; 359: 378-390. 